demic period) who had hemolysis diagnosed $>48$ hours after undergoing hemodialysis. To identify case-patients and to determine background rates, the medical records of patients from facilities $\mathrm{A}, \mathrm{B}$, and $\mathrm{C}$ who were undergoing hemodialysis during the epidemic and pre-epidemic (that is, May 5-19, 1998) periods were reviewed. Experiments simulating hemodialysis with the same lot numbers of hemodialysis blood-tubing cartridge sets used on case- and control-patients were conducted.

The rates of hemolysis among patients at facilities $\mathrm{A}$, $\mathrm{B}$, and $\mathrm{C}$ were significantly higher during the epidemic than the pre-epidemic period $(13 / 118$ vs $0 / 118, P<.001$; $12 / 298$ vs $0 / 298, P=.001$; and $5 / 62$ vs $0 / 65, P=.03$, respectively). All case-patients had hemolysis. Twenty (66\%) had hypertension, $18(60 \%)$ had abdominal pain, and 10 (36\%) were admitted to an ICU. There were two deaths. The only commonality among the three outbreaks was the use of the same lot of disposable hemodialysis bloodtubing from one manufacturer. Examination of the implicated hemodialysis blood-tubing cartridge sets revealed narrowing of an aperture through which blood was pumped before entering the dialyzers. In vitro experiments with the hemodialysis blood tubing revealed that hemolysis was caused by increased pressure on erythrocytes as they passed through the partially occluded hemodialysis blood tubing.

The investigation traced the multiple hemolysis outbreaks to partially occluded hemodialysis blood tubing produced by a single manufacturer. On May 25, 1998, the manufacturer issued a voluntary nationwide recall of the implicated lots of hemodialysis blood-tubing cartridge sets.

FROM: Duffy R, Tomashek K, Spangenberg M, Spry L, Dwyer D, Safranek TJ, et al. Multistate outbreak of hemolysis in hemodialysis patients traced to faulty blood tubing sets. Kidney Int 2000;57:1668-1674.

\section{Modeling Biofilm Antimicrobial Resistance}

In the past 20 years, there has been a great deal of research on biofilms, the slime layers that are deposited on surfaces by microorganisms growing in liquids ranging from water to blood. The organisms are protected by the matrix of the biofilm, and they are, in essence, resistant to germicides or antibiotics.

In a recent paper, Dodds and coinvestigators, from the Center for Biofilm Engineering, Department of Chemical Engineering, Montana State University, in Bozeman, described a computer model capable of integrating mechanisms of biofilm resistance to disinfection by antimicrobial agents. Resistance mechanisms considered included retarded penetration due to a stoichiometric reaction between the antimicrobial agent and biomass, incomplete penetration due to a catalytic reaction between the antimicrobial agent and the biomass, and the existence of a fraction of the cells in a resistant phenotypic state. Mathematical models of these processes were derived and solved in a computer simulation package. Four sets of fitted experimental data on the disinfection of Pseudomonas aeruginosa biofilms were fit to each of the three models. No one model fit all of the data sets adequately. Killing of a 2-day old biofilm by tobramycin was best described by the physiological limitation model. Killing by hypochlorite was best described by the stoichiometric transport model. Killing by hydrogen peroxide was best simulated by the catalytic transport model.

These results suggest that multiple mechanisms of biofilm reduced susceptibility are manifested even in biofilms of the same species and that the particular resistance mechanism depends on the biofilm age, antimicrobial agent, and biofilm thickness. The models presented in this article may be useful for diagnosing mechanisms of biofilm resistance from experimental data.

FROM: Dodds MG, Grobe, KJ, Steward PS. Modeling biofilm antimicrobial resistance. Biotechnol Bioeng 2000;68:456-465.

\section{Aged Dialyzers Cause Outbreak of Severe Reactions}

An event in which seven patients at one hospital developed decreased vision and hearing, conjunctivitis, headache, and other severe neurological symptoms 7 to 24 hours after hemodialysis drew attention to the issue of the long-term integrity of dialysis machines and materials. Hutter and colleagues, from the FDA's Center for Devices and Radiological Health, and the CDC's Hospital Infections Program conducted an investigation to determine the cause of the adverse reactions that occurred during this event. A retrospective cohort study was conducted of all nine patients who received hemodialysis at hospital A on September 18, 1996, the day of the outbreak. A case-patient was defined as any hospital A patient with acute onset of decreased vision and hearing and conjunctivitis after dialysis on that day. Non-casepatients were all others who underwent dialysis at hospital $\mathrm{A}$ on that day but did not develop adverse reactions. In an attempt to reproduce the conditions of the event, cellulose acetate dialysis membranes of various ages were retrieved from other sources and tested for physical and chemical degradation, and degradation products were identified, characterized, and injected intravenously into rabbits. The primary outcome measures were clinical signs and symptoms, time to resolution of symptoms, mortality, and dialyzer type and age, for case- versus non-case-patients.

Seven of the nine patients met the case definition. In addition to diminished vision and hearing, conjunctivitis, and headache, some case-patients had blood-leak alarm activation $(n=6)$, confusion/lethargy $(n=5)$, corneal opacification $(n=4)$, cardiac arrest $(n=2)$, or other neurological signs and symptoms. One case-patient died during hospitalization after the event; five of seven case-patients died within 13 months. Resolution of signs and symptoms varied but persisted more than 3 years or until death in three 